\title{
Parâmetros biométricos de fruto, endocarpo e semente de butiazeiro
}

\author{
Biometrical parameters of fruit, endocarp and seed of pindo palm
}

\author{
Fabrício de Araújo Pedron ${ }^{1}$ Josiane Pacheco Menezes $^{2}$ \\ Nilson Lemos de Menezes ${ }^{3}$
}

\section{- NOTA -}

\section{RESUMO}

\begin{abstract}
O butiazeiro (Butia capitata (Mart.) Becc.) ocorre naturalmente no sul do Brasil. Esta espécie possui potenciais ecológicos, ornamentais e industriais escassamente explorados. Informações sobre variações biométricas de tamanho e peso de frutos e sementes, bem como sua correlação, podem fornecer subsídios para seleção de sementes com maior potencial de germinação e vigor. Os objetivos deste trabalho foram determinar as variações de tamanho e peso de frutos, endocarpos e sementes botânicas de butiazeiro e suas correlações. Foi possível observar a existência de alta correlação, linear e positiva entre o peso e o tamanho de frutos e endocarpos. As sementes de maior peso foram encontradas nos endocarpos que continham duas unidades.
\end{abstract}

Palavras-chave: morfologia de sementes e frutos, biometria, características biométricas.

\section{ABSTRACT}

The pindo palm (Butia capitata (Mart.) Becc.) occurs naturally in the south of Brazil. This specie has a high ecological, ornamental and industrial potential that have not yet been explored. Information about biometrical variations of size and weight of fruits and seeds, as well as their correlations, may support a selection of seeds with higher germination and vigor. The objectives of this study were to quantify fruit, endocarp and botanical seed size and weight variations of pindo palm and their correlation. There was a high, positive correlation between weight and size of fruits and endocarps. Endocarps with two seeds had the highest medium seed weight.

Key words: seed and fruit morphology, biometry, biometrical characteristics.
O butiazeiro (Butia capitata (Mart.) Becc.) é uma planta encontrada, naturalmente, no sul do Brasil (REITZ et al., 1988). O fruto é uma drupa comestível com maturação no período de dezembro a março. Segundo BROSCHAT (1998), o fruto possui mesocarpo carnoso e fibroso e endocarpo duro e denso, contendo de uma a três sementes, apresentando três pontuações dispostas em triângulo denominadas poros de germinação (LORENZI, 1996).

O butiazeiro é uma espécie com potenciais econômico, ecológico e ornamental, no entanto, muito pouco se conhece sobre sua multiplicação, efetuada através de sementes que podem requerer dois anos para concluir o processo de germinação. A distinção das sementes por peso e tamanho pode ser uma maneira de aprimorar os lotes em relação à uniformidade de emergência e vigor das plântulas (ANDRADE et al., 1996; CARVALHO \& NAKAGAWA, 2000).

Este trabalho teve por objetivo obter informações sobre as relações biométricas entre frutos inteiros, endocarpos e sementes botânicas de butiazeiro. Para tanto, 300 frutos foram coletados de dez indivíduos de Butia capitata existentes no Jardim Botânico da Universidade Federal de Santa Maria, quando se encontravam em estádio de dispersão, caracterizando sua maturidade. Inicialmente, foram obtidos o peso e o diâmetro (tamanho) dos frutos

\footnotetext{
${ }^{1}$ Acadêmico do Curso de Agronomia da Universidade Federal de Santa Maria (UFSM), Bolsista PIBIC/CNPq Rua Visconde de Pelotas 1729, apto. 46, 97015-140, Santa Maria, RS. E-mail: fapedron@yahoo.com Autor para correspondência ${ }^{2}$ Biólogo, Mestrando do Programa de Pós-graduação em Agronomia, UFSM.

${ }^{3}$ Engenheiro Agrônomo, Doutor, Professor Adjunto, Departamento de Fitotecnia, Centro de Ciências Rurais, UFSM
} 
inteiros e dos endocarpos; em seguida, os endocarpos foram rompidos, após secagem a sombra por 24 horas, para contagem e pesagem das sementes. Desta forma, foi possível relacionar o número de sementes/fruto com o diâmetro dos endocarpos e frutos e com o peso das sementes. Os dados referentes ao tamanho das sementes não foram utilizados devido ao formato irregular das mesmas dificultarem a padronização da medida, como por exemplo, o diâmetro. Assim, não foi possível realizar a correlação direta entre tamanho de fruto e semente, utilizando-se para isso o parâmetro peso. Foram determinados os valores de mínimo, de máximo, da média, da mediana, do desvio padrão e do coeficiente de variação para os dados obtidos. Adicionalmente, foi realizada análise de correlação entre as variáveis estudadas.

Houve variação nos valores de peso e tamanho de frutos e endocarpos (Tabela 1), principalmente para o peso dos frutos e endocarpos (coeficientes de variação de $39 \%$ e $43 \%$, respectivamente). As variáveis observadas apresentaram correlação alta, linear e positiva entre si com os seguintes valores: peso e tamanho de frutos $\left(r^{2}=0,95\right)$; peso e tamanho de endocarpos $\left(r^{2}=0,94\right)$; peso de frutos e endocarpos $\left(r^{2}=0,95\right)$; tamanho de frutos e endocarpos $\left(r^{2}=0,87\right)$. Os valores de correlação indicam a possibilidade de realizar a coleta de frutos maiores com o objetivo de selecionar endocarpos maiores, na tentativa de se obter melhor desempenho no processo de germinação, como foram obtidos por ANDRADE et al. (1996) e MARTINS et al. (2000), ambos também trabalhando com palmeiras. Foi verificado que endocarpos de maior diâmetro apresentaram maior quantidade de sementes (Tabela 1). Para o peso de sementes, o maior valor médio ocorre nos endocarpos que apresentam duas unidades em seu interior. Existem variações biométricas entre peso e tamanho de frutos, endocarpos e número e peso de sementes de butiá, sendo o diâmetro do fruto um indicador adequado para a coleta de endocarpos maiores, com sementes mais leves e em maior número, ou, endocarpos menores, com sementes mais pesadas, mas em menor número.

\section{REFERÊNCIAS BIBLIOGRÁFICAS}

ANDRADE, A.C.S.; VENTURI, S.; PAULILO, M.T.S. Efeito do tamanho das sementes de Euterpe edulis Mart. Sobre a emergência e crescimento inicial. Revista Brasileira de Sementes, Campinas, v.18, n.2, p.225-231, 1996.

BROSCHAT, T.K. Endocarp removal enhances Butia capitata (Mart.) Becc. (Pindo Palm) seed germination. HortTechnology, Alexandria, v.8, n.4, p.586-587, 1998.

CARVAlHO, N.M.; NAKAGAWA, J. Sementes: ciência, tecnologia e produção. 4.ed. Jaboticabal : FUNEP, 2000. $588 \mathrm{p}$

LORENZI, H. Palmeiras no Brasil: exóticas e nativas. Nova Odessa, SP : Plantarum, 1996. 320p.

MARTINS, C.C. et al. Influência do peso das sementes de palmito - vermelho (Euterpe espiritosantensis Fernandes) na porcentagem e na velocidade de germinação. Revista Brasileira de Sementes, Campinas, v.22, n.1, p.47-53, 2000.

REITZ, P.; KLEIN, R.M.; REIS, A. Projeto madeira do RS. Porto Alegre : Sudesul/HBR, 1988. 528p.

Tabela 1 - Peso e diâmetro de frutos e endocarpos frescos; diâmetro dos endocarpos e peso das sementes segundo o número de sementes por fruto de butiazeiro (Butia capitata). Santa Maria, RS, 2002.

\begin{tabular}{|c|c|c|c|c|c|c|}
\hline Determinações & Mínimo & Máximo & Média & Mediana & Desvio padrão & $\begin{array}{l}\text { Coeficiente de } \\
\text { variação }\end{array}$ \\
\hline Peso $(\mathrm{g})$ dos frutos & 5,627 & 26,428 & 14,379 & 13,055 & 5,63 & $39 \%$ \\
\hline Diâmetro $(\mathrm{cm})$ dos frutos & 1,35 & 4,10 & 3,08 & 3,05 & 0,52 & $16 \%$ \\
\hline Peso (g) dos endocarpos & 0,671 & 4,328 & 2,145 & 1,927 & 0,94 & $43 \%$ \\
\hline Diâmetro $(\mathrm{cm})$ dos endocarpos & 1,00 & 1,90 & 1,41 & 1,40 & 0,22 & $18 \%$ \\
\hline $\mathrm{N}^{\mathrm{o}}$ de sementes/fruto & \multicolumn{6}{|c|}{ Diâmetro $(\mathrm{cm})$ dos endocarpos } \\
\hline 1 & 1,0 & 1,6 & 1,1 & 1,1 & 0,13 & $11 \%$ \\
\hline 2 & 1,1 & 1,7 & 1,6 & 1,6 & 0,11 & $7 \%$ \\
\hline 3 & 1,2 & 1,9 & 1,7 & 1,7 & 0,12 & $7 \%$ \\
\hline $\mathrm{N}^{\mathrm{o}}$ de sementes/fruto & \multicolumn{6}{|c|}{ Peso (g) das sementes } \\
\hline 1 & 0,152 & 0,437 & 0,258 & 0,250 & 0,05 & $18 \%$ \\
\hline 2 & 0,142 & 0,416 & 0,304 & 0,318 & 0,07 & $22 \%$ \\
\hline 3 & 0,089 & 0,345 & 0,252 & 0,261 & 0,05 & $21 \%$ \\
\hline
\end{tabular}

